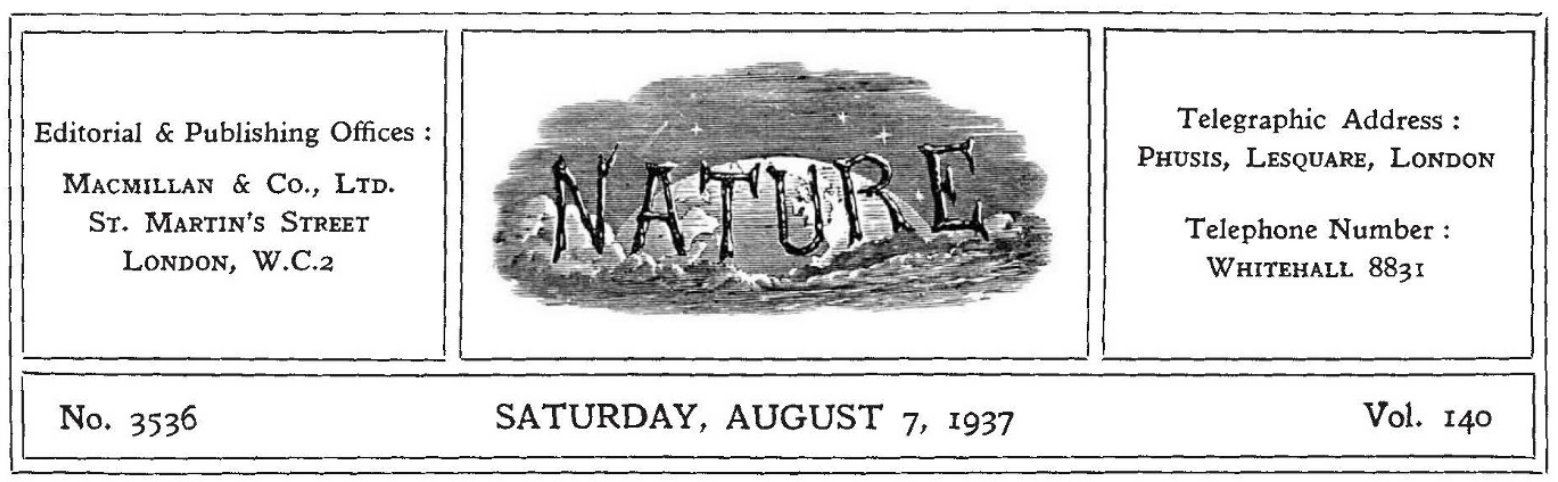

\title{
Systematics in Relation to General Biology
}

$\mathrm{T}^{\mathrm{H}}$ $\mathrm{HE}$ classification of living things is of prime importance, and fundamental to any biological research. Taxonomy, the law of order, is not only one of the oldest branches of biology, but. also remains one that is basic to such more recently developed branches as cytology, ecology, and genetics. This fact cannot be too much stressed. It is somewhat unfortunate that a really satisfactory history of plant and animal taxonomy remains unwritten. The philosophical and logical principles underlying classification have also not received the consideration they deserve, and taxonomists themselves are often ignorant of the real implications of the working hypotheses they adopt. They are often also none too clear as to exactly what is implied by certain words and phrases, such as 'phylogeny' and 'a natural system', which have perhaps assumed a 'blessedness' they scarcely deserve.

Text-books of systematic biology, with very few exceptions, still define the aim of a natural classification as the reconstruction of the course of evolution, and seem to envisage the possibility of eventually producing a schematic tree on which each group hangs on its appropriate branchlet. That this conception rests on a confusion of thought has been suggested by many biologists, and its continued unqualified statement in taxonomic text-books is to be regretted. Perhaps the fullest discussion of the difficulties of equating a natural with a phylogenetic classification is contained in the late Dr. F. A. Bather's presidential address to the Geological Society in 1927, and the same point was stressed recently by Mr. J. S. L. Gilmour in his article "A Taxonomic Problem" in NATURE of June 19, p. 1040. Clear thinking on this fundamental issue is one of the most urgent needs for the future progress of taxonomic work. The older, 'orthodox', taxonomy is based mainly on morphology (including, especially for some groups, anatomy) and with the supplementary use of geographical data, notably in higher animals. This use of morphological criteria as the basis of classification has yielded remarkably valuable results, of which taxonomists may well be proud. There is also no doubt that much yet remains to be done along the well-recognized lines of morphological description and classification, especially in many groups of invertebrates and lower cryptogams and among the faunas and floras of relatively little-explored parts of the world. It will further be generally allowed by taxonomists that there is still room for improvement in their methods of comparative description, terminology, and details of classification without involving farreaching changes.

Within the last two decades, however, there has not only been an almost startling advance in cytology, ecology, genetics, and other sub-sciences of biology, but it has also become increasingly recognized that many discoveries classified under these heads have a bearing on orthodox taxonomy. This impact of newer methods on the still essentially morphological systems of naming and arranging animals and plants can no longer be ignored. It concerns all biologists, whatever may be their particular fields of research. There is no general agreement as to whether new subsidiary systems, with their own terminology and symbolism, should be developed as independently as possible of existing taxonomy, or whether the latter can be so expanded as to include those data of cytology, ecology, genetics, etc., that can be used as 'characters' for arranging organisms into groups. 
Orthodox taxonomy has developed International Rules of Nomenclature. The use of these within their proper field is not disputed, but they may, in their present form, prove a hindrance if rigidly applied to an expanded taxonomy. The possibility and desirability of developing an all-inclusive taxonomy towards an ideal state of perfection needs full investigation.

Existing taxonomy is based on grouping animals and plants into taxonomic units termed varieties, subspecies, species, genera, families, etc. There is, however, considerable disagreement, even among specialists within one group, as to the limits of the units. A general survey of the systematic categories in different groups, a classification of the criteria used, and a careful consideration of recently discovered cytological and genetical facts might well lead to obtaining greater precision, more logical arrangement, and greater uniformity in taxonomy.

Taxonomic literature is very large, scattered in many periodicals, and very diverse in presentation and contents. Museums, herbaria and other institutions contain quantities of material and data, available to the biologist, but often not in a readily accessible form for any particular piece of research. There is, indeed, an almost unrealizable mass of material and of known facts which would yield results of the greatest biological importance if only they were collated and correlated with the rest of biology. It is to be anticipated that new generalizations of considerable importance will emerge in the process. Some of the preliminary analysis has been done, but the results are scattered and uncorrelated; the synthesis would seem to await another Darwin. The task, however, is now beyond the physical powers of any one individual.

While taxonomy is at present based mainly on morphology, its history shows that attempts have been made from time to time to incorporate new ideas. Thus, though taxonomy long antedates the general acceptance of the evolutionary principle, this had in time considerable effects on taxonomic theory and practice. Similarly, especially at the species level, peculiarities of distribution have been utilized in drawing limits between groups.

of recent years, it has become increasingly evident that speciation is neither invariably a simple, clear-cut phenomenon nor due to any one cause or series of causes: rather, a number of factors, internal and external, operate in producing speciation and other evolutionary processes. Their relative importance in different groups needs intensive and extensive investigation. Again, many relevant facts have been published, but until the scattered data have been extracted, correlated and classified, it is impossible to tell along what lines and with what material new research is most likely to throw light on those questions of equal importance to the taxonomist, cytologist, ecologist, and geneticist. It is certain, however, that a great deal of new research is necessary before the problems even of speciation can be solved. The development of biology along synthetic lines does not mean the abandonment of analytical methods in special research. It means rather their intensification, but with a co-ordination at present largely lacking. The collection of material for the eventual publication of a British Fauna and a British Flora on uniform and broad biological lines should serve as a stimulus to research and as a focus for co-ordination.

Many kinds of biological research involve the recording and interpretation of large masses of facts. With proper help and advice, valuable work can be done by local societies, amateurs, schools and colleges. Such research, properly conducted, can have a twofold value : observations or experiments can be made over a wide area or for a long period of time; and they are of very considerable educational value to the persons making them.

Taxonomists, and indeed other biologists, frequently complain that their subject is not well taught in universities and schools. There is much truth in this complaint, and the modification of teaching methods and syllabuses is an urgent need. Students not infrequently know much about the anatomy of a plant or animal without knowing anything about it as a living organism or its relationships to other organisms. The revivification of taxonomy by expanding its scope to include data from other branches of biology and the realization of what they can gain from taxonomy would in itself tend to encourage greater attention to its careful teaching.

That the time is ripe for increased co-operation between workers in different branches of biology will, it is hoped, be acknowledged. In this connexion the recent formation of an "Association for the Study of Systematics in Relation to General Biology", an account of which was published in NATURE of July 24, p. 163, is of special interest. The work of the Association should do much to stimulate interest and co-operation in the various aspects of the impact of modern biological thought on the problems of taxonomy. 